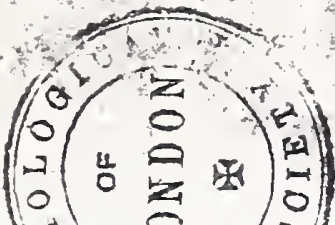 \\ QUAEDAM DEAERA, ET SPEOIES \\ U. 4 \&

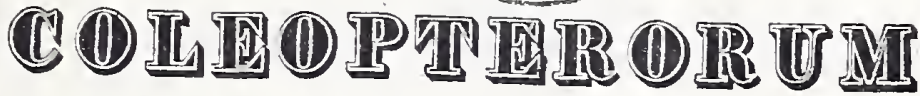 \\ ARCHIDUCATUS AUSTRIAE
}

NONDUM DESCRIPTORUI.

Guilielmo Feallenbucher,

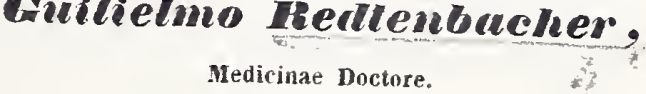

\author{
Dissestatio inaigguralis
}
auctore

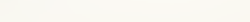


E COL Ra 


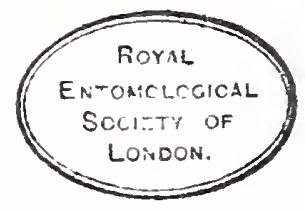

\section{ENTOHOLOGIS AUSTRIACIS.}





\section{Iecturis Salutem.}

In reperiendo libelli academici argumento hand multum dubius haesi, com de Coleopteris; amplo insectorun ordine, quaedam proponere anińus me admoneret. A prinis enim stndionm initiis, versus Historiae maturalis studium exatrsi, atque ex vasto hujns scientiae campo Entomologiam selegi, ut horis subcisivis deliciis atyne recreationi mihi inserviret; hinc cum fratre dilectissimo Lndovico haud temem snperstruxi collectionem Coleopterornm Archiducatus Anstriae; in quo tamen continuo Fanmae uostratis cultu, amicormm aliquot oculatissimorum diligentia nos aptissime adjurat. Itaque muc e re fore existimari, ut quaedan genera atque specimina, in Provincia nostrat degentia, partim dintius cognita sed nondun descripta, partim mperrime reperta enumerarem atque describerem.

Attamen $n o n$ prins tractaminulum hoc publici juris facere andeo, antequam jucundissimo cognato D. Josepho Knoerlein, cujus amore a puero janjam Entomologiae studimm et elementa didiceram, piissimas gratias egerim.

Quo fate temues lae pagellae, nt anicis monumento, entomologis rero usni sint, candidissimms optat 



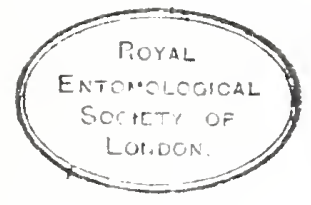

\section{Elmphrus Unriehii Dejcun.}

(1)

blongus, viridi-cuprens, punctatissimns, thorace foveolato, elytris mamillis cupreo - acneis, circulo smaragdino impresso einctis, triplici serie, foreisque smaragdinis ad marginem lat ralem, femoribus snbtus tibiisque testaceis.

$$
\text { *) Long. 31/2-33/4". Lat. 11/2." }
$$

Statura et summa affuitas E. littoralis, sed pauIo major, colore et defectu foveolate in medio lateris thoracis praecipue distiactus. Caput magnum, viridicupreum, nitidum, confertissime punctatum, fronte aequali subconvexa; oculi magni, globosi, brmmei, prominentes. Antennae totae lirete viridi-aeneac. Thorax capite cum oculis antice angustior, in medio ampliatus, postice sinuato-angustatus, angulis posticis rectis, supra convexus, inaequalis, crebre punctatus, virescenti - cupreus, ante apicem carimblablonga, laevis, cupreo-nitida, dein fovea transversil, arcuatis, abbreviata, in suleum profundun longitudinalem, postice subbifidum continuata. ald anguhum posticum foveat majuscula impressa. Scntellum minutum. triangulare. cupreum. Elytra ovata, bassi thorace multo latiora, in medio vịx dilatata, apice rotumdata, supra comexa, cupreo-aenea, undique confertissime punctulatia: in singulo series tres e maculis orbiculatis, violiccis, plerisque in medio papillatis, acneo-cupreis, nitidis,

") Longitudo et latitudo assimnatae sunt in mensura Vienuensi; pollex Viennensis ad dublecim lineats. 


$$
\text { - }
$$


6

laevibus, circulo smaragdino impresso cinctis; intra circulos cujuslibet seriei lineolae longitudinales, elevatiores, laeves, obsoletae et ad marginem lateralem maculae smaragdinae 8 impressae. Corpus subtus viridicupreum, nitidum, punctulatum, abdomine caeruleoviridescente. Femora supra viridi-aenea, subtus testacea, tibiae testaceae, geniculis, apice, tarsisque viridi - aeneis.

Habitat ad litora arenosa fluviorum. A Domino Ullrich mihi communicatus.

\section{Pterostichus Justusii. Spitzy.}

Oblongus, niger, supra coeruleo-micaus, thorace cordato, utrinque biimpresso, elytris oblongis, leviter sulcatis, interstitiis tertio, quinto, septimoque foveolis quadratis irregulariter impressis.

$$
\text { Long.: } 8^{\prime \prime \prime} \text {. Lat.: } 25 / 6 " \text {. }
$$

Pt. fasciato-punctato similis, differt tamen colore, corpore magis depresso atque punctis in elytris. Caput magnum, triangulare, nigrum, nitidum; fronte longitudinaliter bisulcata; Palpi atque antennae nigro - aut rufo-picei. Thorax cordatus, antice posticeque emarginatus, angulis anticis prominulis, acutis, posticis rectis, lateribus marginatus, supra subdepressus, inaequalis, ad apicem arcuatim, in medio canalicula, ad basin utrinque foveola longitudinali majori interna, minori externa, impressus. Elytra oblonga, basi thorace paulo latiora, pone medium parum dilatata, apice singulatim rotundata, subdepressa, coeruleo-micantia, leviter sulcata, interstitiis tertio quinque, quinto sex vel septem, septimoque septem et pluribus foreolis quadratis impressis. Corpus subtus nigrum, aut nigro-piceum, nitidum laeve. Pedes tenues longiusculi, nigri aut nigro-picei, tarsis dilutioribus. 

In alpibns Austriae inferioris Styriae confinibus lectus a D. Spitzy:

Etiam sub nomine Pt. Milleri (Kokeil) mihi communicatus.

\section{Stenolophus liumenalis.}

Oblongus, subdepressus, nigro-piceus, thorace subcordato, utrinque foveolato, foveis impunctatis, elytris simpliciter striatis, macula humerali, ore, antennarum basi, pedibusque pallide-testaceis.

$$
\text { Long.: } 1 \% / 3{ }^{\prime \prime} \text {. Lat.: } 1 / 2 " \prime \text {. }
$$

Statura et summa affinitas St. meridiani: differt tamen figura magis elongata et magis depressa, antennis longioribus, thoraceque in foreis impunctato. Caput magnum, porrectum, nigrum, nitidum. laere, foreola juxta basin antennarum utrinque impressa; labro, mandibulis, palpisque pallide testaceis. Antennae dimidio corporis longiores, basi testaceae, extrorsum fuscescentes. Thorax latitudine medii vix longior, subcordatus, antice emarginatus, angulis anticis deflexis, obtusis, caput arcte cingentibus, lateribus ante medium dilatatis. marginatis; pone medium subsinuatis, angulis posticis subrectis, oblique truncatis, niger, nitidus, laevis, in medio canaliculatus, ante apicem utrinque impressione longitudinale, sublaevi, ad angulum posticum forea profunda, impunctata, impressa. Scutellum minutum, triangulare, nigro-piceum. Elytra oblonga, lateribus parallelis, thoracis basi duplo latiora. apice oblique sinuato-truncata. supra convexa. dorso plaila. nigro-picea, nitida, tenue simpliciter striata: basin macula triangularis pallide testacea, suturam fere attingens occupat; sutura atque margo lateralis fuscescentes. Corpus subtus nigro-piceum, nitidum, laere. Pedes pallide testacei.

rarius lectus.

Sub lapidibus in locis humidis tempore veruali 



\section{IIaliplus ater.}

Ovalis, convexus, ater, nitidus, elytris profunde punctato-striatis, ore, antennis, tibiis, tarsísque rufo-testaceis.

Long. $11 / 2 "$. Lat. $5 / 0^{\prime \prime \prime}$.

Statura et summa affinitas H. impressi. - Caput oblongum, angustum, atrum, nitidum, labro, mandibulis, palpis, antennisque rufo-testaceis; oculi magni, globosi, nigri. Thorax antice multo angustior, late emarginatus, angulis anticis deflexis, productis, acuminatis, lateribus obliquis, rectis, tenue marginatis, postice bi-sinuatus, in medio productus, supra modice convexus, totus ater, nitidus, punctatus, intra basin striga transversa, sinuata, e punctis parvis, obsoletis impressa, intra marginem baseos utrinque rudimentum foreolae obliquae. Elytra thorace vix latiora, dein dilatata, orata, apice oblique truncata, subacuminata, supra convexa, atra, valde nitida, profunde striato-punctata, interstitiis punctis paucis impressis. Corpus subtus atrum, nitidnm, rarius, sed profunde punctatum. l'edes rufo-picei, tibiis, tarsisque dilutioribus.

Semel lectus atque mihi communicatus a $\mathbf{D}$. II a m pe.

\section{Tuthrobium longicone.}

Nigrum, nitidum, punctatum, capite sub-orbiculato, thorace oblongo, linea mediasub-elevata laevi, clytris longitudine thoracis, crebre, punctatis, rubris, basi nigris, antemuis capite cum thorace longioribus, tarsisque rufo-piceis.

$$
\text { Long. } 31 / 2^{\prime \prime} \text { - Lat. } 2 / 3 " \text {. }
$$



Caput sub-orbiculatum, thorace paulo latius, nigrum, nitidum, supra leviter convexum, crebre punctatum, punctis in media fronte rarioribus, parce griseopubescens; palpi brunnei, mandibulae et labrum rufopicea. Antennae capite cum thorace multo longiores, graciles, rufo-piceae, pubescentes, articulo tertio secundo paulo longiore, reliquis secundo aequalibus, sat elongatis, obconicis, ultimo ovato acuminato. Thorax coleopteris multo angustior, latitudine tertia parte longior, lateribus parallelis, angulis rotundatis, supraleviter convexus, parce griseo-pubescens, crebre punctatus, linea media sub-elevata laevi. Scutellum obsolete punctulatum. Elytra thorace vix longiora, dense punctata, griseo-pubescentia, rubra, basi nigra. Abdomen confertissime punctulatum, dense, ast subtiliter griseopubescens, subnitidum, segmentorum ultimi atque penultini marginibus, anoque rufo-piceis. Pedes nigri, rufopubescentes, tarsis rufo-piceis aut ferrugineis. - Mas abdominis segmento inferiore quinto late emarginato, sexto longitudilaliter bi-carinato, carinis in angulum positis, ante apicem conjunctis nigro-pubescentibus, interstitio angusto, impresso, punctulatissimo, apice triangulariter exciso.

Femina abdominis segmenti sexti inferioris apice rotundato.

DD. Comesa Ferrari et $\mathbf{K r a c k}$ owizer hoc mihi communicarunt Coleopteron.

\section{Nematodes strepens.}

Lineari-elongatus, subcylindricus, confertim rugoso-punctatus, holosericeo - pubescens, elytris obsolete punctato-striatis, interstitiis coriaceis, palpis, antennis (in mare flabellatis, in femina pectinatis) tibiis tarsisque fermgineis. 



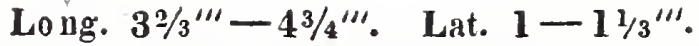

Caput rotundum, deflexum, anterius angustatum, confertissime punctatum, tenue pubescens, vertice convexo; clypeus obverse triangularis, usque ad insertionem antennarum elevate marginatus, rugosus; palpi pallide ferruginei. Antennae longitudine thoracis, ferrugineae, pubescentes; maris flabellatae, articulo priIno magno, elongato, obconico, secundo minimo, globoso, tertio primo breviori, sed crassiori, obconico, reliquis parvis, nodosis, ad faciem exteriorem ramis longis instructis, flabellum formantibus: feminae pectinatae, articulo primo magno, elongato obconico, secundo minimo globoso, tertio primo aequali, reliquis brevibus aequalibus, pectinatis, ultimo elongato ovato. Thorax sub-cylindricus, antice parum angustatus, angulis anticis valde deflexis, lateribus deflexis marginatis, basi bi-sinuatus, lobo medio emarginato, angulis posticis acutis, supra convexus, confertissime punctatus, tenue flavo-pubescens, totus niger, opacus, in medio profunde canaliculatus, canalicula apicem vix attingente. Scutellum parvum, oratum, depressum, punctulatum. Elytra basi thorace vix latiora, elongata, versus apicem sensim angustiora, postice rotundata, supra convexa, dorso planiuscula, nigra, opaca, tenue holosericeo - pubescentia, obsolete striato-punctata, interstitiis granulis minimis confertissine tectis. Corpus subtus nigro-piceum, subnitidum, creberrime punctulatum, holosericeo-pubescens. Pedes tenues, pubescentes, femoribus posterioribus piceis, anticis, tibiis. tursisque dilutioribus, tarsuum articulo primo longissimo, secundo et tertio brevioribus, quarto brevissimo, unguiculari penaltimo duplo longiori.

Rarius lectus a D. H a 10 pe, qui etiam nomen proposuit ob strepitum peculiarem ab imsecto volante calusatum. 



\section{Hiater fulvus.}

Genus. Ampedus, Megerle.

Totus ferrugineus, pubescens, thorace convexo, angulis posticis prominulis, acutis, elytris punctato-striatis, interstitiis punctulatis.

\section{Long. $3^{\prime \prime \prime}$. Lat: $1^{\prime \prime \prime}$.}

Caput parrum, fronte rotundata, convexa, creberrime punctulata, oculis nigris; antennae fere longitudine dimidii corporis, filiformes, articulo secundo et tertio parvis, aequalibus, obconicis. Thorax convexus, subnitidus, fulvo-pubescens, latitudine baseos brevior, anterius angustior, lateribus basique anguste marginatus, angulis posticis prominulis, validis, acutis, carinatis, crebre ast subtilissime punctulatus. Scutellum ovatum, depressum. Elytra basi latitudine thoracis, lateribus subparallelis, versus apicem sensim attenuata, apice rotundata, supra parum convexa, punctatostriatta, interstitiis planis, crebre punctatis, tota fulva, nitida, pubescentia. Corpus subtus saturatius ferrngineum, crebre punctulatum, pubescens. Pedes mediocres, rufo-testacei.

\section{Elater lugens.}

Gen. Sericosomus (Serville) nigritus? Megerle.

Lineari-elongatus, niger, opacus, pube tenui nigra obtectus, thorace ruguloso-punctato, angulis posticis acutis, prolongatis, carinatis, elytris punctatostriatis, interstitiis rugoso-punctatis, tibiis, tarsisque rufo-piceis.

$$
\text { Long. 4-5"'. Lat. 1-11/3". }
$$

Caput parsum, nigrum, subnitidum, creberrime- 

punctatum, fronte subconvexa, margine antico reflexo; ore, palpisque nigro-piceis. Antennae capite cum thorace paulo longiores, nigrae, profunde serratae, articulo primo magno obconico, secundo et tertio minimis globosis, reliquis primo longitudine aequalibus, triangularibus, ultimo ovato. Thorax latitudine dimidio longior, antice angustatus, angulis anticis deflexis, rotundatis, lateribus parum sinuatis, anguste marginatis, angulis posticis acutis, valde productis carinatis, supra convexus, niger, opacus, confertissime rugoso-pumctulatus, in medio bascos rudimentum canaliculac. Ścntellum ovatum, depressum, nigrum, opacum, confertissime rugoso-punctatum. Elytra latitudine thoracis, elongata, apicem versus attenuata, apice rotundata, dorso plana, opaca, pube tenui obtecta, punctato-striata, interstitiis rugoso-punctatis. Corpus subtus nigrum, subnitidum, tenue pubescens, crebre punctatum. Pedes tenues, nigro-picei, tibiis, tarsisque dilutioribus.

\section{Elater" saldonenems. Ziegler.}

Gen. Ectimus. Eschscholl:.

Elongatus, nigro-aeneus, subnitidns, punctatissimus, thorace elongato, antice angustiori, postice canaliculato, elytris obsolete striatis, interstitiis coriaceis, tibiis tarsisque piceis.

$$
\text { Long.: } 41 / 2 " \prime \text {. Lat.: } 11 / 3^{\prime \prime \prime} \text {. }
$$

Caput parvum, prominulum, nigro-aeneum, crebre punctatum, fronte convexa. antice in clypum emarginatum parum producta. Antennae thorace vix longiores, nigrae, sub-serratae, anticulo secundo et tertio sub-aequalibus, nodosis, reliruis elongatis, triangularibus. Thorax latitudine dimidio longior, antice angustior, parnm emarginatus, lateribus anguste marginatis, sub-sinuatis, angulis posticis carinatis, validis, acu- 

tis, elongatis prominulis, supra valde convexus, pulvinatus, nigro-aeneus, subnitidus, tenue pubescens, crebre punctulatus, basi lineola impressa canaliculatus, canalicula in dorso valde obsoleta. Scutellum nigro-aeneum, cordatium, depressum, punctulatum, pubescens. Elytra basi thorace vix latiora, sub-linearia, apicem versus attenuata, acuminata, supra modice convexa, aenea, subnitida, obsolete striata, stria suturali profundiori, interstitiis coriaceis. Corpus subtus nigrum, parum aeneo-micans, magis nitidum, punctulatissimum, tenue pubescens. Pedes tenues elongati, nigro-aenei, tibiis tarsisque piceis, linguiculis ferrugineis.

\section{Canthanis nigmipes.}

Lineari - elongata, nigra, nitida, thorace subquadrato, elytris pallidis, ore, antemarumque basi rufo-testaceis.

\section{Long.: $3^{\prime \prime \prime}$. Lat.: $5 / 6 "$.}

Caput depressum, nigrum, nitidum, vix punctulatum: fronte plana; occipite obsolete canaliculato; oculi globosi brumei, mandibulae rufo-testaceae; palpi infuscati. Antennae dimidio corporislongiores, articulis dnobus primis obscure testaceis. Thorax basi latitudine capitis cum oculis, antice parum angustior, subquadratus, latitudine paulo latior, undique elevatomarginatus, angulis omnibus rotundatis, niger, nitidus, disco convexus, inaequalis, subpunctatus. Scutellum triangulare. nigrum. Elytra thorace panlo latiora lineari-elongata, crebre rugoso-punctata, pallide ochracea. Corpus nigrum, nitidum, tenue pubescens. Pedes nigro-picei, flavo-pubescentes, geniculis, tibiarum apice, unguiculisque dilutioribus.

Pluries lecta a D. Krackowizer monte Schneeberg in Urticae floribus. 



\section{Malachius festivus.}

\section{Ger. Anthoconuts. Erichs.}

Lineari-elongatus, obscure viridi-aeneus, ore, antemis, elytrorum nairgine exteriore anguste, apice late, pedibusque flavis.

\section{Long.; 13/4" Lat. : $3 / 4^{\prime \prime \prime}$.}

Statura M. pulicarii. Caput nigro-viridescens, fronte impressa, transversim tri-foveolata; oculis prominulis: clypeo flavescente, labii basi, palporumque maxillarium articulo penultimo testaceis, reliquis infuscatis. Antennae flliformes, longitudine dimidii corporis, testaceae, apicem versus brunnescentes, articulus primus introrsum parum incrassatus, reliquis paulo longior, secundus tertio dimidio brevior, nodosus, tertius et quartus interne vix incrassati, reliqui obconici, aequales. Thorax longitudine latior, sub-quadratus, postice parum angustatus, angulis rotundatis, supra modice convexus, lateribus deflexus, basi leviter transversim impressus, subtilissime punctulatus, rufo-testaceus. Elytra thorace vix latiora, oblonga, convexa, obseure viridi-aenea, margine laterali anguste, fasciaque apicali antrorsum dentata, late flavis; maris elytra sunt retuso - excavata, externe rotundata, interne in unguiculum atrum, arcuatum, erectum producta, excavatione ipsa infuscata. Corpus subtus nigro-aeneum, marginibus segmentorum abdominalium tenue palidis. Pedes pallidi, femorum posticorum basi superne infuscata. Semel captus a D. Krackowizer.

\section{Parnus longives, Parreyss.}

Oblongus, supra fusco-ferrugineus holosericeotomentosus, elytris obsolete punctato-striatis, pedibus longis, fusco-piceis, tibiis, tarsisque fermgineis. 



\section{Long.: 21/3"'-22/3"'. Lat.: 1"'.}

Caput retractum, rotundatum, convexum, creberrime punctatum, nigro-fuscum, ore intra sternum productum occultato. Antennae ante oculos insertae, in fovea profunda frontis immersae, fuscescentes. Thorax antice angustior, longitudine latior, angulis anticis productis, deflexis, acutis, basi bi-sinuatus, lobo medio truncato, angulis posticis acutis; supra valde convexus, pulvinatus, fuscus, holosericeo-micans, crebre, ast subtiliter punctulatus, obsoletissime canaliculatus, canalicula apicem non attingente. Scutellum cordatum, punctulatum. Elytra thoracis basi paulo latiora, oblonga, pone humeros sinuata, anguste marginata, apice sinuato-acuminata, supra convexa, humeris prominulis, fusco-ferruginea, pube densa sericea tecta, obsolete punctato-striata, striarum punctis obsoletis, remotis, interstitiis convexiusculis, crebre, ast-subtiliter granulatis. Corpus subtus ex pube densa grise 0 -micans, granulis obscurioribus crebre tectum. Pedes longi, postici longissimi, longitudine fere totius corporis, femora fusco-picea, tibiis, tarsisque ferrugineis.

D. Parreyss Tauriam, D. Villa in suo catalogo Dalmatiam uti ejus patriam assignant, ast a D. Comite Ferrari etiam in Austria ad rivulos captus.

\section{Genus nov. Phlocostichus.}

Palpi maxillares quatuor articulati, articulo primo minimo, conico, secundo tertioque multo crassioribus, breviter conicis, ultimo ovato, truncato. Palpi labiales tri-articulati, articulo ultimo majore, ovato, apice truncato.

Labrum transversum, rotundatum, apice vix emarginatum.

Labium transversum, margine antico semi-circulari, elevato; dentibus duobus productis distantibus. 

Ligula brevis, quadrata, emarginata, margine ciliato. Mandibulae modice arcuatae, apice bifidae.

Maxillae palpis maxillaribus multo breviores, simplices, rectae, internae, apiceque ciliatae.

\section{Oculi laterales, rotundati, prominuli.}

Antennae articulis tribus ultimis crassioribus ; articulo

primo crasso, transverso, secundo subconico, latitudine duplo longiore, 4. 5. 6. secundo aequalibus, septimo paulo minore, octavo et nono campanulatis, ultimo ovato, acuminato. Abdomen segmentis quinque compositum.

Tarsi quatuor anteriores quinque - postici quatuor articulati, teretes, parum ciliatae.

Affinitatem quod attinet, hoc genus ad familiam Xylophagorum collocandum est.

\section{Phloeostichus denticollis.}

Oblongus, niger, subnitidus, pubescens, punctatus, thorace subquadrato, lateribus dentato, elytris punctato-striatis, fasciis duabus undatis, flavoferrugineis, tibiis, tarsisque fuscis.

$$
\text { Long.: 2"“. Lat.: } 5 / 6^{\prime \prime \prime} \text {. }
$$

Caput triangulare, profunde punctatum, pubescens, uigrum, nitidum; vertice transversim canaliculato, fronte impressa. Antennae longitudine capitis cum thorace. nigrae, pubescentes. Thorax sub-quadratus, angulis anticis oblique truncatis, dente parso armatis; lateribus parmm dilatatis, anguste marginatis, in medio bi- aut tridentatis. angulis posticis rotundatis, subdepressus . crebre et profunde punctatus. tenuissime et obsolete canaliculatus, in dor'so ante basin foveolis duabus, rotundis. approximatis impressus. Scutellum transverse ovatum, nigrum punctulatum. Elytra thorace latiora, oblonga, pubescentia. margi- 

nata, posterius paululum ampliata, dorso plana, profunde punctato-striata, interstitiis planis, subtilissime seriato-punctatis, fasciis duabus flavo-ferrugineis, undatis, anteriori ante medium posita, interrupta, posteriori paulo pone medium, integra, abbreviata, suturam et marginem non attingente. Corpus subtus nigrum, nitidum, sub-pubescens, punctatum. Pedes nigropicei, pubescentes, tibiis, tarsisque dilutioribus. -

Ipse legi sub cortice aceris pseudoplatani non penitus emortui in Alpibus Aust. superioris.

Dominus Villa Mediolanensis hoc insectmm asservavit in sua collectione sub nomine: Ips denticollis.

\section{Margus oloscurus.}

Oblongus, subtus fusco-ferrugineus, supra rufoaut nigro-piceus, opacus, crebre et profunde punctatus, thorace transverso, elytris elevato-striatis, interstitiis tri-seriatim punctatis, palpis, antennis, pedibusque rufo-ferrugineis.

$$
\text { Long.: 2"'. Lat.: } 3 / 4^{\prime \prime \prime} \text {. }
$$

Statura et summa affinitas $\boldsymbol{M}$. ferruginei, differt tamen magnitudiıe, figura latiori, colore, thoraceque transverso, ante medium dilatato. Caput sub-orbiculatum, porrectum, nigrum, crebre punctatum, clypeo lato porrecto, in medio emarginato, rufo-piceo, palpi laete ferruginei. Antennae capite cum thorace breviores, ferrugineae, apice dilutiores, articulo primo et secundo sub-aequalibus, tertio paulo majoribus, reliquis gradatim incrassatis, tribus ultimis abrupte majoribus. Thorax transversus, angulis anticis obtusis, deflexis, lateribus ante medium dilatatis, postice angustatis, angulis posticis sub-rectis, lateribus, basique tenue marginatus, basi sub-sinnatus, supra leviter convexus, ae- 

qualis, niger, opacus, crebre punctatus, punctis in medio rarioribus. Scutellum triangulare, nigrum, punctatum. Elytra basi thoracis latiora, lateribus subparallelis, apice rotupdata, supra modice convexa, nigra, opaca, elevato-striata, striis versus suturam fere evanescentibus, interstitiis subtiliter seriato-punctatis, seriebus punctorum trinis, una in medio interstitii singuli, reliquis ad striam elevatam positis. Corpus subtus rufo-piceum, non tam crebre, ast profunde punctatum. Pedes mediocres laete ferruginei, tibiae anticae margine anteriori non crenatae. -

\section{Cistela sulfuripes. Dahl.}

Elongata, angusta, nigra, nitida, elytris obsolete striatis, interstitiis coriaceis, pedibus pallide testaceis.

\section{Long.: $21 / 2 " 1$. Lat.: $1^{\prime \prime \prime}$.}

Caput porrectum, elongatum, versus os angustatum, punctulatum, fronte inter autennas impressa, palpis, mandibulisque modo testaceis modo obscure fuscis; oculi prominuli brunnei. Antennae dimidio corpore vix longiores, filifornes, nigrae aut nigro-piceae. Thorax brevis, transversus, angulis anticis deflexis, obtusis, lateribus tenue marginitus, ante medium dilatatus, angulis posticis subrectis, supra modice convexus, niger, tenue pubescens, opacus, subtilissime coriaceus. Scutellum triangulare, nigrum, nitidum, punctulatum. Elytra thorace paulo latiora, elongata, postice paulo attenuata, apice singulatim rotundata, supra conrexa, nigra, subnitida, pubescentia, striata, striis obsoletis, ad marginem exteriorem fere evanescentibus, interstitiis subtiliter coriaceis. Corpus subtus nigrum, nitidum, crebre punctulatum. Pedes elongati, pallide testacei; postici, tarsique obscuriores. 



\section{Phytonomus maculatus. Dahl.}

Hypera Helluigii. Megerle.

Ovatus, apterus, niger, púbe densa, depressa, fusco-cinerea variegatus, thorace latitudine longiori, punctato, elytris connatis, punctato-striatis, interstitiis alternis maculis nigris denudatis, irregulariter signatis.

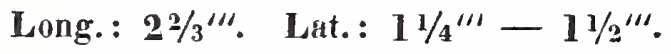

Caput breve, convexum, nigrum, punctulatum, cinero - pubescens, fronte inter oculos impressa; oculi depressi, nigri; rostrum thorace brevius, subcylindricum, crassum, vix arcuatum, punctulatum, pubescens. Antennae elytra fere attingentes, ferrugineae, clava obscuriori, pubescentes, articulo primo et secundo funiculi obconicis, aequalibus, reliquos tertia parte longitudinis superantibus, clava ovata, acuminata. Thorax latitudine medii longior, lateribus rotundato-dilatatus, supra modice convexus, niger, crebre punctatus, pube densa, fusco-cinerea variegatus, linea media angusta, ex pilis albido-griseis congesta: Scutellum minutum, pilosum. Elytra in ipsa basi thorace parum latiora, dein valde dilatata, apice rotundata, concreta, supra convexa, nigra, remote punctato-striata, tota squamulis piliformibus depressis aut cinëreis aut fuscis variegata, interstitiis alternis irregulariter nigro-maculatis, maculis pilis fere destitutis. Corpus subtus nigrum, punctulatum, pube cinerea tectum. Pedes rufo-picei aut picei, dense cincreo-pubescentes. Mas multo angustior, magis elongatus; Femina magis ovata magisque in medio dilatata.

Habitat in Verbasco phlomoide. 



\section{1\%. Otiorlnymehus mandibularis.}

Ovatus, piceus, fusco-cinereo-squamosus, thorace rufo-piceo, tuberculato, tuberculis rubro-pellucidis, elytris punctato-striatis, squamulis fuscocinereis variegatus, interstitiis seriatim granulatis, seriatimque setosis, mandibulis valde elongatis.

$$
\text { Long.: } 2 \% 3^{\prime \prime \prime} \text {. Lat. : } 11 \frac{1}{3}{ }^{\prime \prime} \text {. }
$$

Caput porrectum, nigrum, occipite sublaevi, glabro, fronte rugulosa, squamulis cinereis adspersa; oculi nigri, prominuli ; rostrum longitudine capitis, piceum, rugosum, cinereo-squamulosum, in medio canaliculatum, ad latera bifoveolatum; mandibulae piceae, porrectae, longitudine fere dimidii scapi, arcuatae. Antennae vix longitudine dimidii corporis, crassiusculae, fusco-piceae, cinereo-pubescentes, scapo funiculoque inter se longituline aequalibus, articulo primo et secundo funiculi subaequalibus, obconicis, elongatis, reliquis monili-formibus, clava ovata, acuminata, pube densa obtecta. Thorax globosus, basi, apiceque truncatus, lateribus rotundato-dilatatus, supra valde convexus, rufo-piceus, crebre tubercalatus, tuberculis rubro-pellucidis, pube rara, cinerea tectus. Scutellum nullum. Elytra ovata, basi thoracis latitudine, pone basin dilatata, lateribus abdomen arcte includentibus, dorso antice plana, postice valde declivia, picea, squamulis fusco-cinereis variegata, punctato-striata, interstitiis tuberculosis, tuberculis in seriem positis, setulisque cinereis, suberectis instructis. Corpus subtus piceum, punctulatum, griseo-pubescens. Pedes longiusculi, dense cinereo-pubescentes, rufo-picei aut ferruginei, femoribus clavatis, anticis dente obtuso armatis, posterioribus muticis.

Unicum exemplar captum a J). Miller. 



\section{Hylesinus suturalis.}

Brevis, niger, opacus, thorace transverso, ruguloso-punctato, elytris punctato-striatis, interstitiis granulatis, atureo-setosis, setis ad suturan condensatis, antennis pedibusque obscure ferrugineis.

\section{Long.: $1^{\prime \prime \prime}$. Lat.: $2 / 3^{\prime \prime \prime}$.}

Caput breve, vix porrectum, nigrum, crebre punctulatum; fronte impressa subtilius punctulata, linea media elevata; oculi oblongi nigri; rostrum brevissimum, capite parum angustius, nigrum, mandibulis validis atris, nitidis. Antennae capite paulo longiores, ferugineae, clava articulata, magna. Thorax longitudine latior, antice angustior, vertici aequalis, basi sinuatus, angulis rotundatis, supra convexus, niger, ad basim simpliciter, ad apicem ruguloso-punctulatus, pube ferruginea parce adspersus, linea media longitudinali obsoleta, apicem non attingente, et impressionibus duabus, absoletis, transversis, curvatis ad marginem baseos. Scutellum minutum, rotundatum, nigrum, punctatum. Elytra thoracis basi vix latiora, antice singulitim, postice sumptim lotundata, supra convexa, nigra, punctatostriata, interstitiis granulatis, granulis setis rigidis aureis obsessis, sutura densius setosa, interstitio secundo in medio laevi subsetoso. Corpus subtus nigrum, nitidum, fortiler punctatum. Pedes breviusculi, obscure ferruginei, tibiae apice dilatatae, externe crenatae.

Bis solummodo captum hoc insectum distinctum a D. Prester prima et memet ipso altera vice.

\section{Geuus nov. Hinopalocerus.}

Palpi maxillares 4 articulati, articulo primo conico, secundo et tertio brevibus, transversis, fuarto tres 

praecedentes longitudine superante, lato, ovato, apice truncato.

Palpi labiales 3 articulati, articulo primo et secundo minimis, tertio magno, ovato, apice trincato.

Labrum transversum, leviter emarginatum.

Labium corneum, angustum, profunde emarginatum. Ligula brevis, paraglossis magnis, longo-ciliatis. Mandibulae modice arcuatae, apice bifidae, intra apicem dente obtaso armatae.

Maxillae bifidae, lobo externo oblongo-quadrato, apice ciliato, lobo interno angusto, semi-membranaceo, externo breviore, apice ciliato.

Oculi laterales, parum prominuli.

Antennae sub-moniliformes, articulo ultimo majore, 10articulatae, articulo primo crasso, elongato, obconico, secundo sub-cylindrico, paulo breviori, tenuiore, ante basin constricto, tertio iterum minori sub-sequentibus fere aequali, etiam constricto 4., 5., 6., 7., 8., 9. globosis, ultimo valde incrassato, latitudine paulo longiore, obconico, apice rotundato; articulus quilibet, setis paucis inclinatis ornatus.

Tarsi 4-articulati, articulo primo minimo, secundo et tertio aequalibus, brevibus, quarto elongato, praecedentibus sumptim multo longiore, arcuato, unguiculis duobus simplicibus.

Etiam ad familiam Xylophagorum, reponendum est hoc genus.

\section{MInopalocenus setosus.}

Oblongo-ovatus, fusco-ferrugineus, thorace quadrato, scabro, in medio canaliculato, elytris oblongo-ovatis, sulcatis, interstitiis, seriebus tuberculorum, seta brevi grisea auctorum, per paria approximatis, instructis. 



\section{Long.: $11 \frac{1}{2}{ }^{\prime \prime \prime}$. Lat.: $2 / 3^{\prime \prime \prime}$.}

Caput quadrangulare, scabrum, fronte excavata, clypeo lato, transverso, antice vix emarginato. Antennae longitudine capitis cum thorace, ferrngineac, setosae. Thorax quadratus, supra convexus, angulis anticis acutis, lateribus rectis, crenulatis, posticis rotundatis, supra verrucis seta brevi, rigida obsessis scaber, in medio fovea longitudinali exaratus. Scutellum rotundum, minutum, in medio impressum, obscure fuscum. Elytra oblongo-ovata, dimidio thorace latiora, ferruginea, opaca, sulcata, interstitiis elevatis, seriebus tuberculorum seta brevi grisea auctorum, per paria approximatis, instructis, marginali et suturali siugulis. Corpus subtus rufo-ferruginenm, crebrius et profunde punctatum, setulisque obtectum, segmentorum abdominalium apicibus marginatis. Pedes validi, mediocres, ferruginei, setosi.

Rarius lectus a D. II ampe sub cortice aceris campestris.

In diversis collectionihus jam existit sub nomine ,Sarrotrium rufum $“$. M e gerle.

\section{Minotoma quadil- foveolatum.}

Flavo - ferrugineun, pubescens, thoracis disco quadri - foveolato, elytris seriatim -punctatis seriatimque pilosis.

$$
\text { Long.: 1"'. Lat.: 1/3"'. }
$$

Caput sub-quadratum. rugoso-punctatum, flavo-pubescens, fronte obsolete longitudiualiter bi-impressa; oculi nigri. Antennae capite paulo longiores, articulis duobus primis magnis, primo subquadrato, secundo paulo minore, globoso, articulo ultimo magno, primum crassitie superante, ovato. - Thorax quidratus, láte- 



\section{4}

ribus rectis, marginatis, angulis anticis reclis, leviter rotundatis, angulis posticis sub-rectis, basi parum rotundatus, supra sub-depressus, in disco foveolis quatuor latis sat profundis, contiguis impressus. Scutellum minutissimum, punctiforme. Elytra marginata, basi thorace paulo latiora, in medio vix dilatata, apice truncata, angulo marginali rotundato, anum non tegentia, seriatim punctata, punctis pilis depressis, flavis obsessis, interstitiis glabris, sublaevibus. Corpus subtus ferrugineum, crebre punctulatum, flavo-pubescens. Pedes breves, pubescentes, rufo-ferruginei.

\section{Callidium scabricolle. Megerle.}

Lineari-elongatum, subdepressum, tenue pubescens, supra nigro- aut rufo-piceum, subtus ferruginemn, thorace subquadrato, in medio sublaevi, lateribus transversim unicato, elytris rugoso-punctatis, striis obsoletis, elevatis, tribus, antennis obscurius, palpis pedibusque dilutius ferrugineis aut testaceis.

$$
\text { Long. : } 3 \frac{1 / 2}{5} 5^{\prime \prime \prime} \text {. Lat. : } 1-1 \frac{1 / 2}{\prime \prime \prime} \text {. }
$$

Caput majusculum, rotundatum, nigrum, nitidum, pilis tectum, in medio parcius, pone oculos confertim punctulatum, fronte inter antennas impressa. Oculi brunnei, integri. Antennae longitudine dimidii corporis, filiformes, obscure ferrugineae, scabrae, pilosae. Thorax subquadratus, latitudine parum longior, lateribus subrectis, antice posticeque rotundatus, subdepressus, pilosus, ad basin obsolete canaliculatus, dorso lateribus profundiori, punctato, nitido, linea media sublaevi, laferibus transversim muricatus, opacus, basi anguste marginatus. Scutellum ovatum, nigrum, nitidum; ovatum. Elytra basi thorace parum 

latiora, postea sensim dilatata, apice singulatim rotundata, subdepressa, nigro-picea, basi dilutiora, creberrime ruguloso-punctata, pubescentia, lineis elevatis tribus, obsoletis. Corpus subtus ferrugineum, nitidum, crebre punctatum, pilosum, thorace subtus, segmentorumque abdominalium margine obscuriore. Pedes breves, tenues, pallide ferruginei, pilosi.

A. D. Comite F errari ad describendum benevole communicatum.

\section{Saperia uncinata.}

Gen. Phyloecia. Dejean.

Lineari-elongata, nigra, griseo-pubescens , thorace ruditer punctato, longe piloso, simulque pube cinerea in lineam mediam et lateralem condensata adsperso, scutello albido-pubescente, elytris punctatis, segmentorum abdominalium primo atque secundo apice uncinatis.

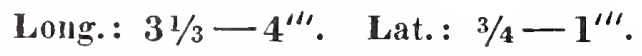

Caput thorace latius, nigrum punctulatum, pube cinereo-albida adspersum, simulque longe pilosum, fronte convexiuscula; oculi magni, nigro-brunnei, profunde emarginati. Antennae vix longitudine corporis, setaceae, nigrae, sub-pilosae. Thorax sub-cylindricus, in medio vix dilatatus, intra basin et apieem leviter constrictus, crebre punctatus, niger, longe pilosus, pubeque cinerea adspersus, in lineam mediam et lateralem, apicen non attingentes, condensata. Scutellum semi-ovale, nigrum, dense albido-pubescens. Elytra thorace fere duplo latiora, humeris elevatis, linearia, postice parum angustiora, apice singulatim rotundata, supra in dorso plana, nigra, anterius pilosa, profunde punctata, pube tenui, cinerea adspersa. Corpus subtus nigrum, cinereo-pubescens, thorace subtus crebre et profunde, pectore abdomineque ra- 

vius atque subtilius punctulatis, segmentoram abdominalium primum et secundum in medio apice in uncos producta. Pedes mediocres, nigri, cinereo-pubescentes, femoribus simplicibus.

Videtur marem atque feminam his uncis esse instructos.

Bis lecta a fratre meo Ludovico in loco Brigittenau.

\section{Saperda biguttata. Megerle.}

Gen. Phyloecia. (Dej.) venusta, Fridwaldsky.

Brevior, nigra, pilosa, thoracis linea media, scutello, elytrorum macuila apicali, pectoris lateribus, segmentorumque abdominalium marginibus albido-pubescentibus, pedibus testaceis.

$$
\text { Long.: } 31 \frac{1 / 2}{\prime \prime \prime} \text {. Lat.: } \mathbf{1}^{\prime \prime \prime} \text {. }
$$

Caput thorace latius, nigrum, pilosum punctulatum, fronte planiuscula, pube grisea tecta. Oculi nigri, emarginati. Antennae longitudine corporis, setaceae, nigrae, sub-pilosae. Thorax cylindricus, lateribus perparum ampliatus, niger, pilosus, punctatus, linea media albido-pubescente. Scutellum exacte quadratum, albido-pubescens. Elytra thorace duplo latiora, oblonga, postice parum angustiora, convexa, dorso planiuscula, nigra, pilosa, crebre et profunde punctata, ante apicem utrinque guttula albido-pubescente ornata. Corpus subtus nigrum, subnitidum, tenue pubescens, subtilissime coriaceun, pectoris lateribus linea albida longitudinali signatis, segmentorum abdominalium marginibus pube albida annulatis. Pedes omnes flavotestacei.

Semel lecta a D. U1lrich circa Linz. 



\section{Inalica circumndata.}

\section{Gen. Balanomorpha, Cheorolat.}

Oblongo-ovata, nigro-aenea, nitida, elytris antennis, tibiis tarsisque testaceis, sutura apiceque elytrorum nigricantibus.

\section{Long. $11 / 4^{\prime \prime \prime}$. Lat. $2 / 3^{\prime \prime \prime}$.}

Statura et summa affinitas H. affinis; differt tamen colore thoracis, margineque laterali elytrorum fuscescente. Caput triangulare, nigrum, nitidum, vertice et fronte rare punctatis, non tuberculatis; oculi prominuli. Antennae longitndine dimidii corporis, basi pallide testaceae, extrorsum fuscescentes. Thorax transversus, antice angustior, postice latior, lateribus valde dellexis, obliquis, subrectis, anguste marginatus, basi in medio productus, angulis rotundatis, supra parum convexus, nigro-aeneus, marginibus interdum ferrigineopellucidis, ad latera fortius, in dorso subtilius punctulatus. Scutellum parvum triangulare, nigrum, nitidum, laeve. Elytra antice thorace paulo latiora, in medio dilatata, postice sensim attenuata, apice rotundata, supra modice convexa, livide testacea, seriato-punctata, sntura et apice triangulariter nigris, margine laterali plus minusve infuscato. Corpus subtus nigro-aeneum, nitidum, punctulatum. Pedes quatuor anteriores tenues, femoribus piceis, apice, tibiis tarsisque testaceis; femora postica magna, nigro-aenea. - In campis aridis circa Viennam rarius lecta.

\section{IInitica nigriceps.}

Gen. Teinodactyla, Cherrolat.

Ovata, nigra, nitida, subtilissime punctulatia. 

thorace, elytris, antennarum basi pedibusque pallide testaceis, sutura nigricante.

$$
\text { Long. } 5 / 6^{\prime \prime \prime} \text {. Lat. } 1 / 2 " \text {. }
$$

Medium occupat quoad staturam et magnitudinem inter H. Nasturtii et pusillam. Caput triangulare, nigrum aut piceum, sublaeve, fronte tuberculata. Antennae dimidio corpore longiores, basi pallide testaceae, extrorsum fuscescentes, gradatim crassiores, pubescentes; oculi magni, prominuli, nigri. Thorax parvus, transversus, antice truncatus, postice rotundatus, antice posticeque vix-ad latera distincte marginatus, lateribus rotundatis, supra convexiusculus, pallide-testa-. ceus, sublaevis. Scutellum triangulare, nigrum, nitidum, laeve. Elytra basi thorace duplo latiora, postea sensim dilatata, orata, supra convexa, dilutius testacea, rare et subtiliter punctulata, sutura saltem in medio late nigro-fusca. Corpus subtus nigrum, nitidum, punctulatum. Pedes omnes pallide testacei, femoribus posticis valde incrassatis, paulo obscurioribus.

\section{Haltica Campanulae.}

Gen. Aphlhona, Chevrolat.

Ovata, nigro-aenea, nitida, supra viridi-aenea, anteunarum basi, pedibusque flavo-testaceis, thorace punctulato, elytris sat fortiter punctatis, angulo suturali obtuso.

$$
\text { Long. 1"'. Lat. 1/2"'. }
$$

Parva, H. Euphorbiae simillima differt tamen colore, punctatura, anguloque suturali obtuso.

Caput triangulare, aeneum, nitidum, subtiliter rugulosum, fronte bi-tuberculata, tenue carinata. Antennae dimidio corpore longiores, articulis quinque baseos laete flavo-testaceis, reliquis gradatim crassioribus 

et obscurioribus, ultimo nigro; acuminato. Thorax brevis, transversus, angulis anticis valde deflexis, lateribus rotundatus, supra modice convexus, virescentiaeneus, nitidus, lateribus fortius, dorso subtilius punctulato. Scutellum mediocre, nigrum, nitidum, sublaeve. Elytra-basi thorace paulo latiora, postea dilatata, apice rotundata, angulo suturali obtuso, supraconvexa, viridi-aenea, nitida, crebre et fortiter punctata. Corpus subtus nigro-aeneum nitidum, punctulatum. Pedes mediocres, flavo-testacei, femoribus posticis clavatis, paulo obscurioribus.

Pluries lecta a D. Comite Ferrarisupra Campanulam rotundifolian. 



\section{Theses defendendae.}

1 .

In individuis, in quibus tuberculosis pulmonum in evolutione versatur, cultrum non adhibendum.

2.

In Syphiliticis operatio cataractae per extractionem non admittatur.

is.

Lux anima, lux mors visus.

4.

Per insitionem variolae vaccinae varicellae non praecaventur.

\section{5.}

Syphilis inveterata et scirrhus difficillime a se invicem distinguuntur.

6.

Tympano perforato auditus non tollitur.

$\%$.

Sanguis et quoad qualitates physicas in morbis varie mutatur

8.

Et talis mutatio sanguinis in causa est, ut tuberculosi vix unquam typho corripiantur.

9.

Omnium doctrinarum medicarum Pharmacologia a dimidio saeculo plurimum neglecta est. 

10.

Historiae naturalis studiosi facultate observandi prae aliis instruuntur.

11.

Natura nusquam major quam in minimis.

12.

Zoologia ut ad gradum perfectionis extollatur, quem Mineralogia occupat, maxima offendens obstacula, anni atque anni non sufficiunt.

13.

Vera species physico-historica in aliam transitum non admittit, hinc in Entomologia paucae existunt verae species.

14.

Plura adhuc dantur Coleoptera, quae simile principium acre praebent ac Cantharides.

15.

Substantia ejaculata Coleopterorum Brachinorum cauterium sistit fortissimum.

16.

Mors individui est vita alterius; materia non perit, sed variat formas, hinc nulla mors. 

